\title{
Arthroscopic Management of Tibial Spine Avulsion in Children: a Retrospective Study with a Minimum Four Year Follow-Up
}

\author{
G. F. Trinchese ${ }^{1}$, A. Oliviero ${ }^{2,3}$, F. Oliva ${ }^{2,3}$, N. Maffulli ${ }^{2-5}$ \\ 1 Department of Orthopaedics and Traumatology, Villa Malta Hospital, Sarno, Salerno, Italy \\ 2 Department of Trauma and Orthopaedic Surgery, AOU San Giovanni di Dio e Ruggi D'Aragona, Salerno, Italy \\ 3 Department of Medicine, Surgery and Dentistry, University of Salerno, Baronissi, Salerno, Italy \\ 4 Queen Mary University of London, Barts and the London School of Medicine and Dentistry, Centre for Sports \\ and Exercise Medicine, Mile End Hospital, London, U.K. \\ ${ }_{5}$ School of Pharmacy and Bioengineering, Keele University School of Medicine, Thornburrow Drive, Stoke on \\ Trent, U.K.
}

\author{
CORRESPONDING AUTHOR: \\ Nicola Maffulli \\ Queen Mary University of London \\ Barts and the London School of Medicine \\ and Dentistry \\ Centre for Sports and Exercise Medicine \\ Mile End Hospital \\ 275 Bancroft Road \\ London E1 4DG, U.K. \\ E-mail:n.maffulli@qmul.ac.uk
}

DOI:

10.32098/mltj.03.2021.15

LEVEL OF EVIDENCE: 4

\begin{abstract}
SUMMARY
Isolated avulsion of the tibial spine occurs in children and can be associated with residual insufficiency of the anterior cruciate ligament (ACL). Completely displaced tibial spine fractures are managed with open or arthroscopic surgical fixation. This retrospective study describes the results obtained with arthroscopic reduction at a mean follow-up of 81.6 months.

Eleven patients were treated with arthroscopic suture fixation, at the last follow-up all patients exhibited a negative Lachman test and a negative pivot shift test, the mean International Knee Documentation Committee (IKDC) score was 97.9 and the mean Lysholm score was 96. All patients showed bone union at plain radiography.

Arthroscopic reduction with suture fixation is a safe and reliable method to treat displaced or comminuted avulsion fractures of the tibial eminence.
\end{abstract}

\section{KEY WORDS}

Tibial fracture; adolescent; arthroscopy; avulsion fractures.

\section{INTRODUCTION}

Isolated avulsion of the tibial spine is relatively frequent, with an incidence of 3 per 100.000 inhabitants per year (1). They usually occur in children, though they are becoming more frequent in adults $(2,3)$. After such injury, the knee become lax given the insufficiency of the anterior cruciate ligament (ACL), and exhibits decrease in range of motion (ROM) (4). Nondisplaced and selected partially displaced fractures can be treated non-operatively, on the other hand completely displaced tibial spine fractures are managed with surgical fixation (5). The aim of surgery includes restoration of knee stability, restoration of the range of motion and return to function (5). Fracture non-union, arthrofibrosis and malunion are the most frequently reported complications of tibial spine fractures (6). The former may lead to limitation in $\mathrm{ROM}$, and the latter produces residual ligament laxity.
Surgery is necessary to prevent these issues, and anatomical reduction with stable fixation is required to restore normal knee biomechanics if the avulsion is completely or partially displaced $(2,7,8)$. Clinical evaluation, computed tomography and magnetic resonance imaging help to identify the fracture patterns and guide the treatment options (9).

Meyers and McKeever $(10,11)$ identified three main types of fractures: Type I (minimally displaced anterior margin); Type II (superior displacement of the anterior edge with an intact posterior hinge); Type III (completely displaced and avulsed tibial eminence). Type III fractures have been sub-classified in Type IIIa (with fragments completely displaced) and type IIIb (fragments are displaced and rotated) $(10,11)$.

Zaricznyj described a fourth type of tibial eminence fracture, with comminution (12). 
Type I fractures are usually treated with the knee immobilization in full extension, while in type II, III and IV fractures treatment options is more controversial. In Type II, III and IV fracture the reduction may not be full, and arthroscopic evaluation and anatomic reduction with internal fixation is technically demanding (13).

The present study evaluates the effectiveness of suture fixation on a series of patients affected by tibial spine fracture.

\section{MATERIALS AND METHODS}

In this retrospective study, eleven patients were treated between 2010 and 2017. The inclusion criteria were: 1) displaced tibial eminence avulsion; 2) anterior knee laxity of grade II or higher. Exclusion criteria were: 1) tibial plateau fracture; 2) nerve or vessel damage; 3 ) multi-ligament injury or knee dislocation. Patients were assessed after the arthroscopic surgery, and comparison with the contralateral uninjured knee was made. The mean follow-up was 81.6 months, range 48-128.

The primary outcome of the study was the reduction in post-operative knee laxity, that was evaluated with the Lachman test and the pivot shift test, and measured by Rolimeter ${ }^{\circledR}$ (Aircast, DJO Global, Vista, CA, USA); secondary outcomes were the improvement in the International Knee Documentation Committee (IKDC) scores, the Lysholm score, and the bone union. Fracture healing was assessed on lateral radiograph, and clinically based on disappearance of anterior knee pain.

All patients gave their written consent before surgery, and the relevant institutional review board approved the study. All patients underwent the same treatment protocol for arthroscopic suture fixation using FiberWire (Arthrex, Naples, FL, USA) sutures to undertake the relevant fixation.

\section{Surgical Technique}

The procedure was performed under spinal anesthesia. Patients were placed in the supine position and a pneumatic tourniquet was inflated after limb exsanguination. Standard anteromedial (AM) and anterolateral (AL) portals were used. Associated meniscal tears or other lesions were assessed and addressed before fracture treatment. In most patients, the transverse intermeniscal ligament was interposed between the superiorly displaced fragment and the tibia, preventing accurate reduction. Using a N.1 PDSII from the AM portal the ligament was pulled anteriorly, thus facilitating reduction of the tibial eminence fragment and its maintenance (figure 1). After a comprehensive arthroscopic examination, a probe was used to lift the avulsed fragment and allow access to the fracture bed. A shaver was used to carefully debride the frac- ture hematoma and remove the smaller fracture fragments to mobilize the avulsed tibial spine (figure 2).

Trial reductions was performed using the probe. The reduced fragment was temporarily fixed with a $1.5 \mathrm{~mm}$ Kirschner wire inserted percutaneously from the superomedial aspect of the knee joint (figure 3). The tip of the tibial ACL guide was placed on the base of the ACL through the anteromedial portal (figure 4). A $1 \mathrm{~cm}$ longitudinal incision was made just medial to the anterior tibial tuberosity and deepened to the tibial surface to place the tibial ACL guide in contact with the tibial cortex. Two $2.4 \mathrm{~mm}$ guide wire were drilled into the joint through the guide from the proximal tibia through the reduced fragment. One guide wire was placed just to the medial aspect and the other just to the lateral aspect to the insertion of the ACL. Next, a $4 \mathrm{~mm}$ cannulated drill bit was used to make the two tibi-

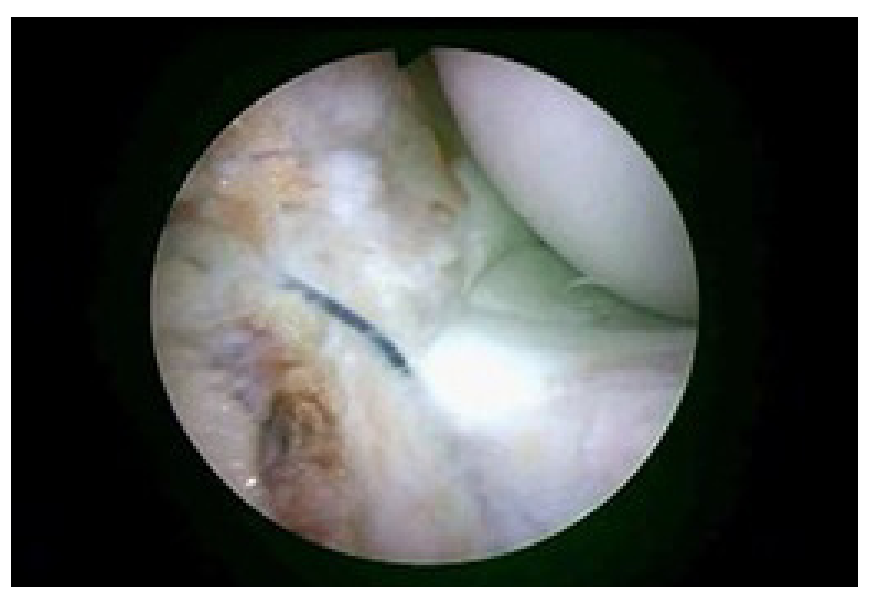

Figure 1. The intermeniscal ligament is pulled anteriorly, thus facilitating reduction of the tibial eminence fragment by using a N.1 PDSIl from the AM portal.

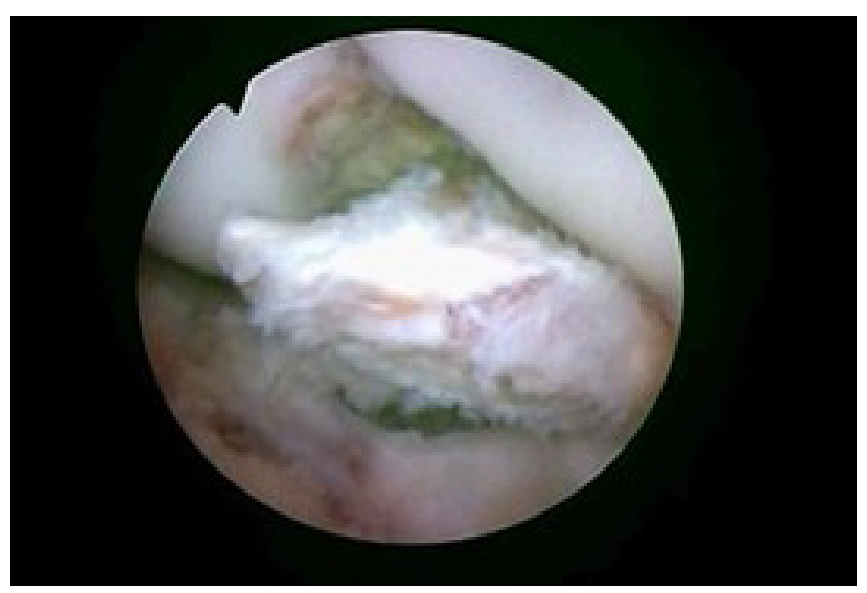

Figure 2. The tibial spine is fully mobilized by removing hematoma and smaller fracture fragments. 


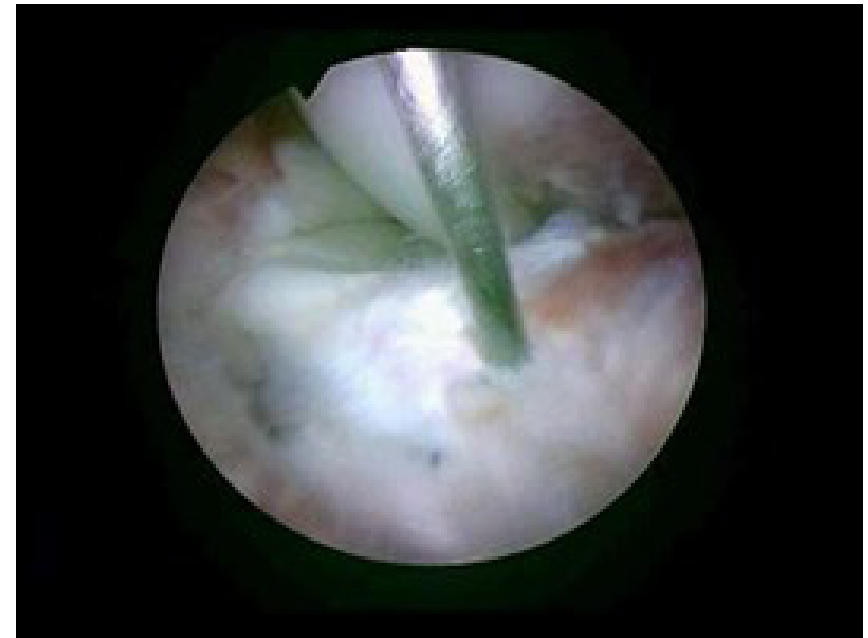

Figure 3. The reduction of the avulsed fragment is achieved, and the tibial spine is temporarily fixed by using a K-wire inserted from the superomedial aspect of the knee joint.

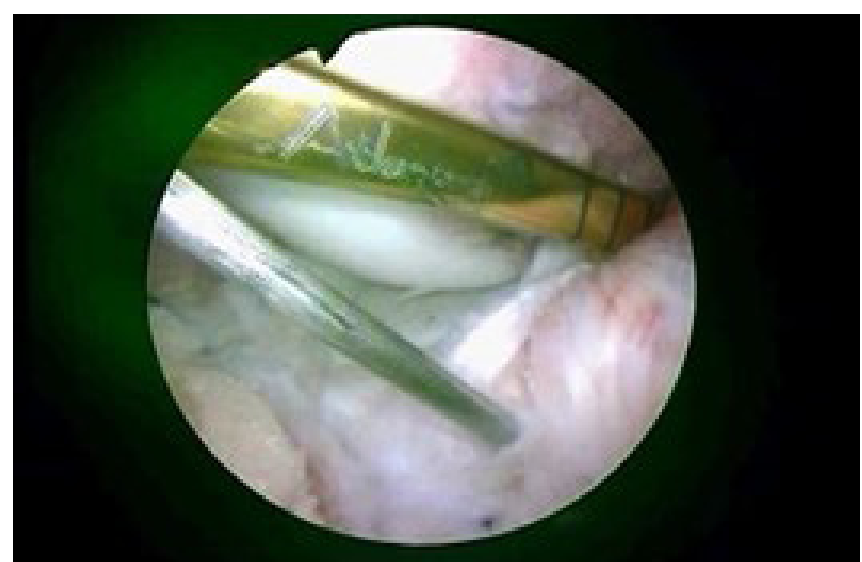

Figure 4. The point of the C-ring drill guide is placed on the base of the ACL through the anteromedial portal.

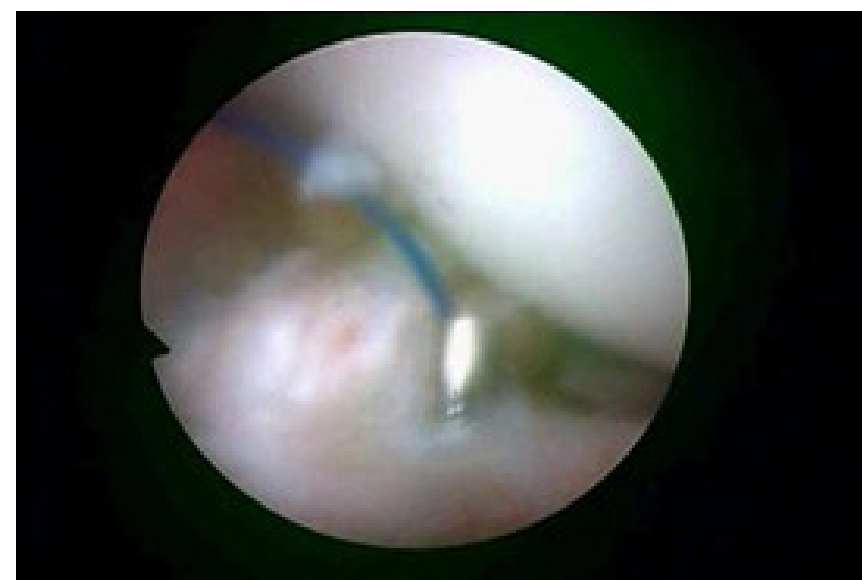

Figure 5. The outer cannulated drill sleeve is left in place after having drilled the $4 \mathrm{~mm}$ tibial tunnel. al tunnel. The surgeon unscrewed and removed the inner drill bit while leaving the outer cannulated drill sleeve in place (figure 5). A $45^{\circ}$ suture hook was passed through the base of the ACL just proximal to its tibial insertion on the fracture fragment, and a N.1 PDSII was shuttled through the base of the ACL (figure 6). One N. 5 FiberWire suture was then inserted into the lateral cannulated drill, retrieved from the anterior portal with a grasper and shuttled, using the PDS loop, through the ACL (figure 7). After this, a PDS loop was inserted into the medial cannulated drill and the FiberWire was passed through the second tunnel, exiting from the anterior aspect of the tibia. The reduction was confirmed visually and tested with the probe (figure 8), and the suture was then tied over the bony bridge on the anterior-medial cortex of the tibia (figure 9).

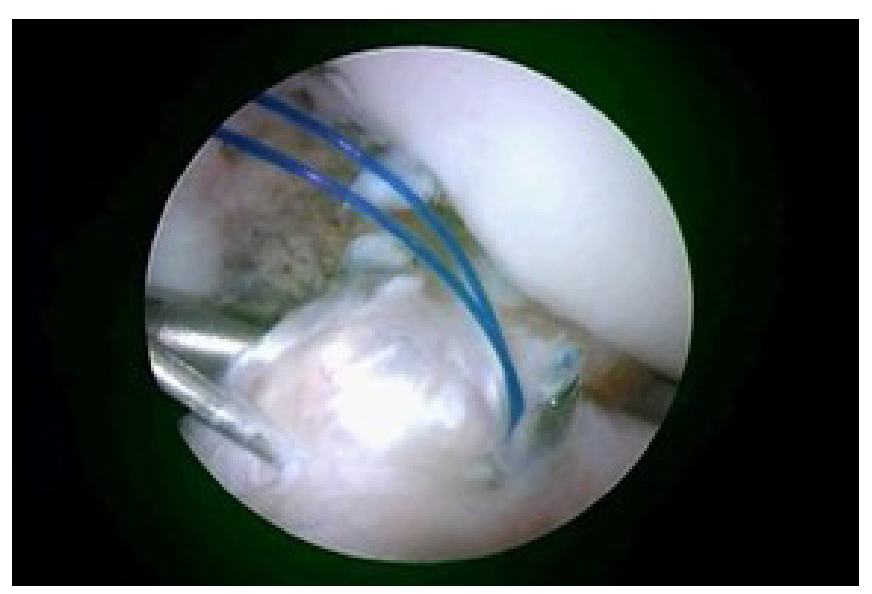

Figure 6. A $45^{\circ}$ suture hook is passed through the base of the $A C L$ just proximal to its tibial insertion site on the fracture fragment and a N.1 PDSII is then shuttled through the base of the ACL.

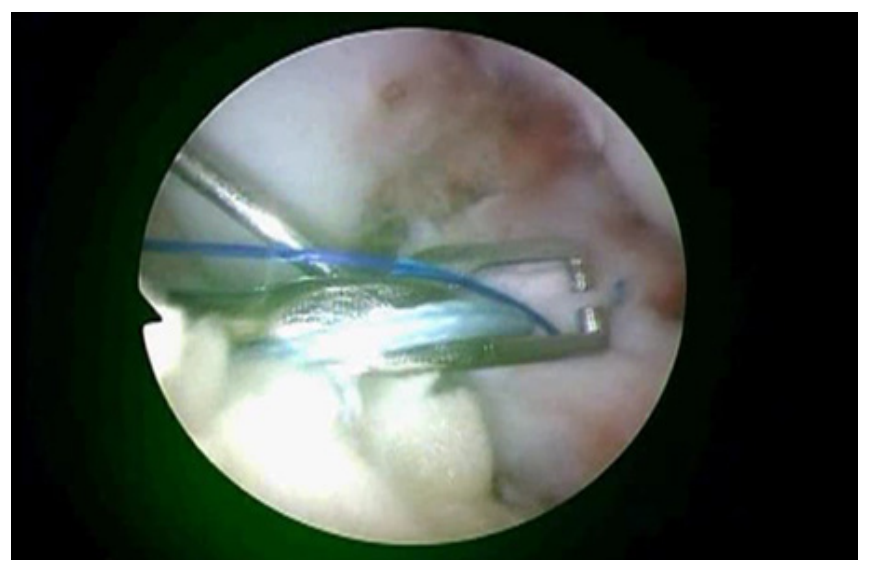

Figure 7. One N.5 FiberWire is inserted into the lateral cannulated drill, retrieved from the anterior portal with a grasper and shuttled, by use of the PDS loop, through the ACL. 


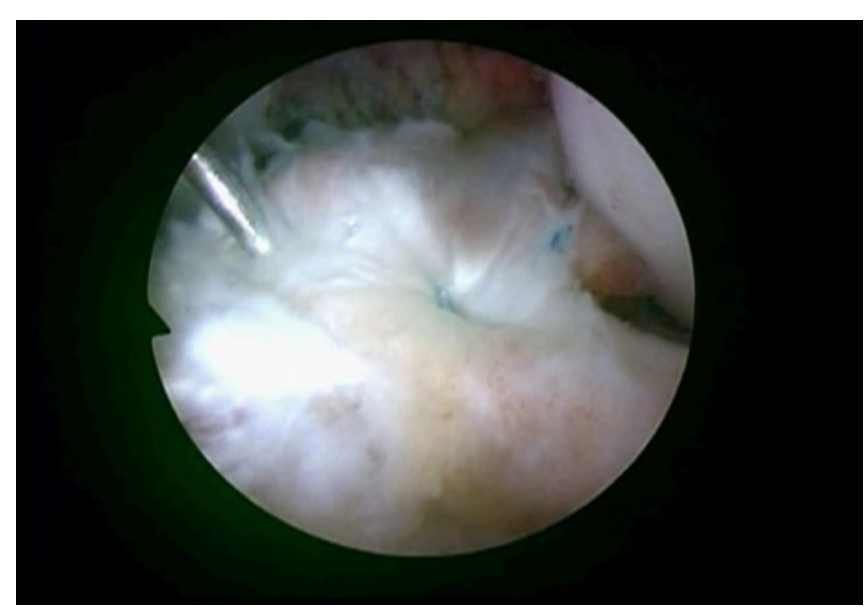

Figure 8. The reduction of the fragment is achieved.

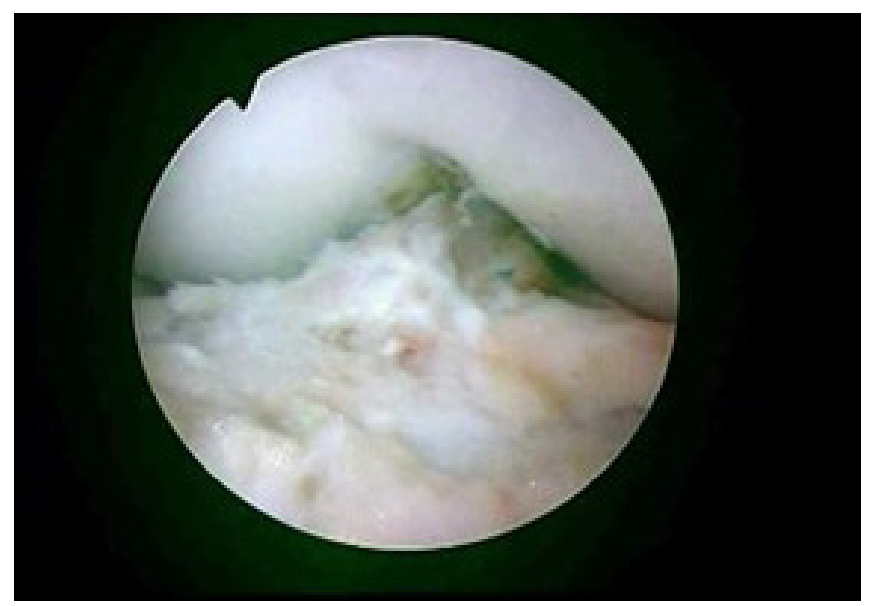

Figure 9. The tibial spine is fixed by tying the sutures over a bony bridge on the anterior-medial cortex of the tibia.

\section{Postoperative protocol}

Different post-operative and rehabilitative protocols are proposed in the literature, based on the surgeon's preferences, the type of fracture, the quality of fixation and patient compliance (14).

Compared to the protocol used by other authors, $(15,16)$ ours provided for faster weight bearing and mobilization. In any case, most of the studies in the literature concord for immediate weight bearing and early mobilization (14).

The knee was immobilized in a brace locked in full extension for 2 weeks. Strengthening exercises and stimulation of quadriceps and hamstrings were started immediately and weight bearing permitted with crutches as tolerated. After 2 weeks the brace was progressively unlocked and mobilization started. The brace was removed after 4 weeks and mobilization continued until the range of motion was completely recovered.

\section{RESULTS}

The main characteristics of the study are summarized in table I. Eleven patients, 9 Males and 2 Female, mean age $18.27 \pm 7.74$ (12-35) were recruited (6 type II, 3 type III and 2 type IV). The mean antero-posterior excursion measured with the Rolimeter was $7.6 \mathrm{~mm}$ for the operated side and $6.2 \mathrm{~mm}$ for the opposite side. All patients exhibited a negative Lachman test and a negative pivot shift test at final follow-up. Seven patients were classified IKDC $A$ and 4 patients IKDC B. The mean IKDC subjective score was 97.9 (range 94 to 100). The mean Lysholm score was 96 (range 90 to 100) at the last follow up. All patients showed bone union at plain radiography. One patient reported to have experienced difficulty in regaining motion post-operatively and underwent an arthroscopic arthrolysis with full recovery. This second procedure consisted of a debridement of arthrofibrotic scar tissue inside the joint. No hardware disturbances have been observed and no other patients required a second operation. No complications were other complications were reported. No infection or venous thrombosis was noted. Of the recruited patients, nine reported no pain during moderate or strenuous activities; two patient reported intermittent and slight pain with moderate or strenuous activities at the last follow-up.

Table I. Main characteristics of the study.

\begin{tabular}{ll}
$\begin{array}{l}\text { Number of patients } \\
\text { male } \\
\text { female }\end{array}$ & 11 \\
\hline Mean age & $2(81.8 \%)$ \\
\hline Mean Follow-up & $18.27 \pm 7.74$ \\
\hline Meyers and McKeever Classification & $\begin{array}{l}81.6 \text { months } \\
\text { (range } 48-128)\end{array}$ \\
& 3 type III \\
\hline Rolimeter & 2 type IV \\
$\quad$ operated side & \\
\hline opposite side & 7.6 mm \\
\hline Lachman test & 6.2 mm \\
\hline Pivot shift & All patients negative \\
\hline Post-op IKDC Classification & ALL patients negative \\
$\quad$ type A & 7 patients \\
type B & 4 patients \\
\hline Mean IKDC score at last follow-up & 97.9 \\
& (range 94 to 100) \\
\hline Mean Lysholm score at & 96 \\
last follow-up & (range 90 to 100) \\
\hline Complication & 1 arthrofibrosis \\
\hline
\end{tabular}




\section{DISCUSSION}

Surgical treatment of displaced intercondylar eminence fractures is essential to prevent nonunion or malunion. Most surgeons recommend surgery for displacement of more than $3 \mathrm{~mm}$ in type II, type III and IV fractures of intercondylar eminence $(12,17,18)$.

A systematic review of the literature has not demonstrated long term superiority of open surgery over arthroscopic surgery or screw versus suture fixation techniques (19). However, recent arthroscopic fixation techniques demonstrated less morbidity and complications, better evaluation and treatment of any associated lesions, and faster functional recovery in comparison to open procedures (2). Arthroscopic fixation techniques include percutaneous Kirschner wire fixation, screw fixation (20), TightRope fixation (21), steel wire fixation or suture fixation (22-24).

In 1982, McLennan first reported the treatment of fracture of the intercondylar eminence of the tibia with Kirschner wire but anterior knee pain, instability and decrease in motion were the main complications (18), with high rates of failure (25).

Recent studies have demonstrated the biomechanical advantages of newer suture-based fixation methods compared to fixation with screws, however the best method of fixation has not been defined as each technique has specific advantages (26).

Although fixation with the screw technique allows several advantages such as immediate loading and early mobilization, it cannot be used in cases of comminution or small fragment size. Furthermore, in case of malpositioning with associated cartilage damage or persistent intolerance, it will be necessary to remove the screw. Finally, growth disturbances may occur in patients with open physis (14).

Suture fixation methods are basically classified into 2 groups. The first involves the direct suture of the ACL fibers, vice versa the other possibility involves the reduction and fixation of the avulsed bone fragment. This last technique is obviously not usable when the tibial spine is comminuted, but in these cases it is possible to suture the base of the ligament itself in order to obtain a solid fixation (27). Ligament suture methods require special instruments to place intraligamentous sutures and a suture passer to thread ligament sutures in the joint. Tsukada et al. found significantly greater anterior translation after cyclic loading in fractures stabilized with pullout suture fixation compared with antegrade screw fixation $(28,29)$. In a cadaveric biomechanical study, specimens fixed with Fiberwire had a mean ultimate strength of $319 \mathrm{~N}$. Those fixed with cannulated screws had a mean ultimate strength of $125 \mathrm{~N}(\mathrm{P}=.0038)$. There was no significant difference between the mean stiffness of FiberWire constructs $(63 \mathrm{~N}$; SD, $50 \mathrm{~N})$ and the mean stiffness of the cannulated screw constructs $(20 \mathrm{~N}$; SD, $32 \mathrm{~N})$. FiberWire fixation of eminence fractures provides biomechanical advantages over cannulated screw fixation, and may influence the type of treatment one chooses for patients with tibial eminence fractures (30). Using absorbable suture fixation, Verdano et al. also showed good and very good results in 21 patients with complete union of the avulsed fragment at 2 years of follow-up without complications $(31,32)$. Commonly used screw methods can easily crush bony bone fragments and cut ligaments, increasing the likelihood of damaging the open physis and requiring a second procedure to remove the implant. In addition, the operative space is limited $(30,33,34)$.

All our patients presented returned to full function after the index surgery. No hardware disturbances were reported, only one patient required a second operation of arthroscopic arthrolysis, and no infection or venous thrombosis were noted. Notwithstanding the small number of patients treated, the suture fixation procedure described allows excellent results for the tibial eminence fracture avulsion. There are several advantages in using the described technique. First, the suture is performed at the base of the ACL and not in the bone, making it easy to reduce even in type III or IV fractures. Furthermore, this type of fixation proved to be biomechanically superior to the technique with screws which could then risk breaking the bone fragment and also require removal in case of intolerance $(27,35,36)$. Finally, in case of difficult reduction can be managed with multiple sutures for precise repair of bone fragments.

\section{CONCLUSIONS}

Displaced or comminuted avulsion fractures of the tibial eminence can be easily managed with arthroscopic reduction with suture fixation allowing to restore ACL length, stabilize fragments, promote early motion, and minimize morbidity. Direct intrarticular inspection allows anatomic reduction and the treatment of further intra-articular pathology. This technique is easily reproducible with low hardware costs compared with sutures using anchors or other hardware, and the cannulated drill significantly simplifies the process of suture passage while maintaining fracture reduction and minimizing surgical steps. The study meets the ethical standards of the journal (37).

\section{CONFLICT OF INTERESTS}

The authors declare that they have no conflict of interests. 


\section{REFERENCES}

1. Hargrove R, Parsons S, Payne R. Anterior tibial spine fracture - an easy fracture to miss. Accid Emerg Nurs 2004;12(3):173-5.

2. Delcogliano A, Chiossi S, Caporaso A, Menghi A, Rinonapoli G. Tibial intercondylar eminence fractures in adults: arthroscopic treatment. Knee Surg Sports Traumatol Arthrosc Off J ESSKA 2003;11(4):255-9.

3. Kendall NS, Hsu SY, Chan KM. Fracture of the tibial spine in adults and children. A review of 31 cases. J Bone Joint Surg Br 1992;74(6):848-52.

4. Ahmad CS, Shubin Stein BE, Jeshuran W, Nercessian OA, Henry JH. Anterior cruciate ligament function after tibial eminence fracture in skeletally mature patients. Am J Sports Med 2001;29(3):339-45.

5. Ganley TJ, Brusalis CM. Surgical Reduction and Fixation of Tibial Spine Fractures in Children: Multiple Fixation Strategies. JBJS Essent Surg Tech 2016;6(2):e18.

6. Vargas B, Lutz N, Dutoit M, Zambelli PY. Nonunion after fracture of the anterior tibial spine: case report and review of the literature. J Pediatr Orthop Part B 2009;18(2):90-2.

7. Lowe J, Chaimsky G, Freedman A, Zion I, Howard C. The anatomy of tibial eminence fractures: arthroscopic observations following failed closed reduction. J Bone Joint Surg Am 2002;84(11):1933-8.

8. W P, T Z, M R. [Fracture of the tibial head]. Unfallchirurg 2006;109(3):219-32; quiz 233.

9. Myer DM, Purnell GJ, Caldwell PE, Pearson SE. ORV Arthroscopic Reduction and Internal Fixation of Tibial Eminence Fractures. Arthrosc Tech 2013;2(4):e341-5.

10. Meyers MH, McKEEVER FM. Fracture of the intercondylar eminence of the tibia. J Bone Joint Surg Am 1959;41$\mathrm{A}(2): 209-20$; discussion 220-2.

11. Meyers MH, McKeever FM. Fracture of the intercondylar eminence of the tibia. J Bone Joint Surg Am 1970;52(8):1677-84.

12. Zaricznyj B. Avulsion fracture of the tibial eminence: treatment by open reduction and pinning. J Bone Joint Surg Am 1977;59(8):1111-4.

13. Lubowitz JH, Elson WS, Guttmann D. Part II: arthroscopic treatment of tibial plateau fractures: intercondylar eminence avulsion fractures. Arthrosc J Arthrosc Relat Surg Off Publ Arthrosc Assoc N Am Int Arthrosc Assoc 2005;21(1):86-92.

14. Osti L, Buda M, Soldati F, Del Buono A, Osti R, Maffulli N. Arthroscopic treatment of tibial eminence fracture: a systematic review of different fixation methods. Br Med Bull 2016;118(1):73-90.

15. Reynders P, Reynders K, Broos P. Pediatric and adolescent tibial eminence fractures: arthroscopic cannulated screw fixation. J Trauma 2002;53(1):49-54.

16. May JH, Levy BA, Guse D, Shah J, Stuart MJ, Dahm DL. ACL tibial spine avulsion: mid-term outcomes and rehabilitation. Orthopedics 2011;34(2):89.

17. Cannamela PC, Quinlan NJ, Maak TG, Adeyemi TF, Aoki SK. Knee Extension Does Not Reliably Reduce Acute Type II Tibial Spine Fractures: MRI Evaluation of Displacement During Extension Versus Resting Flexion. Orthop J Sports Med 2019;7(7):2325967119860066.
18. McLennan JG. The role of arthroscopic surgery in the treatment of fractures of the intercondylar eminence of the tibia. J Bone Joint Surg Br 1982;64(4):477-80.

19. Gans I, Baldwin KD, Ganley TJ. Treatment and Management Outcomes of Tibial Eminence Fractures in Pediatric Patients: A Systematic Review. Am J Sports Med 2014;42(7):1743-50.

20. Wiegand N, Naumov I, Vámhidy L, Nöt LG. Arthroscopic treatment of tibial spine fracture in children with a cannulated Herbert screw. The Knee 2014;21(2):481-5.

21. Faivre B, Benea H, Klouche S, Lespagnol F, Bauer T, Hardy P. An original arthroscopic fixation of adult's tibial eminence fractures using the Tightrope ${ }^{\circledR}$ device: a report of 8 cases and review of literature. The Knee 2014;21(4):833-9.

22. Kluemper CT, Snyder GM, Coats AC, Johnson DL, Mair SD. Arthroscopic suture fixation of tibial eminence fractures. Orthopedics 201336(11):e1401-6.

23. Memisoglu K, Muezzinoglu US, Atmaca H, Sarman H, Kesemenli CC. Arthroscopic fixation with intra-articular button for tibial intercondylar eminence fractures in skeletally immature patients. J Pediatr Orthop Part B 2016;25(1):31-6.

24. Sawyer GA, Hulstyn MJ, Anderson BC, Schiller J. Arthroscopic suture bridge fixation of tibial intercondylar eminence fractures. Arthrosc Tech 2013;2(4):e315-8.

25. Gan Y, Xu D, Ding J, Xu Y. Tension band wire fixation for anterior cruciate ligament avulsion fracture: biomechanical comparison of four fixation techniques. Knee Surg Sports Traumatol Arthrosc Off J ESSKA 2012;20(5):909-15.

26. Doral MN, Bilge O. Editorial Commentary: Arthroscopic Fixation of Tibial Eminence Fractures-Which Technique Is the Best Has Not Been Defined Yet! Arthrosc J Arthrosc Relat Surg Off Publ Arthrosc Assoc N Am Int Arthrosc Assoc 2018;34(5):1617-20.

27. Huang T-W, Hsu K-Y, Cheng C-Y, et al. Arthroscopic suture fixation of tibial eminence avulsion fractures. Arthrosc J Arthrosc Relat Surg Off Publ Arthrosc Assoc N Am Int Arthrosc Assoc 2008;24(11):1232-8.

28. Koukoulias NE, Germanou E, Lola D, Papavasiliou AV, Papastergiou SG. Clinical outcome of arthroscopic suture fixation for tibial eminence fractures in adults. Arthrosc J Arthrosc Relat Surg Off Publ Arthrosc Assoc N Am Int Arthrosc Assoc 2012;28(10):1472-80.

29. Tsukada H, Ishibashi Y, Tsuda E, Hiraga Y, Toh S. A biomechanical comparison of repair techniques for anterior cruciate ligament tibial avulsion fracture under cyclic loading. Arthrosc J Arthrosc Relat Surg Off Publ Arthrosc Assoc N Am Int Arthrosc Assoc 2005;21(10):1197-201.

30. Bong MR, Romero A, Kubiak E, et al. Suture versus screw fixation of displaced tibial eminence fractures: a biomechanical comparison. Arthrosc J Arthrosc Relat Surg Off Publ Arthrosc Assoc N Am Int Arthrosc Assoc 2005;21(10):1172-6.

31. Pan R-Y, Yang J-J, Chang J-H, Shen H-C, Lin L-C, Lian Y-T. Clinical outcome of arthroscopic fixation of anterior tibial eminence avulsion fractures in skeletally mature patients: a 
comparison of suture and screw fixation technique. J Trauma Acute Care Surg 2012;72(2):E88-93.

32. Verdano MA, Pellegrini A, Lunini E, Tonino P, Ceccarelli F. Arthroscopic absorbable suture fixation for tibial spine fractures. Arthrosc Tech 2014;3(1):e45-48.

33. Sommerfeldt DW. Arthroscopically assisted internal fixation of avulsion fractures of the anterior cruciate ligament during childhood and adolescence. Oper Orthopadie Traumatol 2008;20(4-5):310-20.

34. Parr RR. Arthroscopic reduction and headless cannulated compression screw fixation of adults' tibial eminence fractures. Am J Orthop Belle Mead NJ 2006;35(12):558-61.
35. Kogan MG, Marks P, Amendola A. Technique for arthroscopic suture fixation of displaced tibial intercondylar eminence fractures. Arthrosc J Arthrosc Relat Surg Off Publ Arthrosc Assoc N Am Int Arthrosc Assoc 1997;13(3):301-6.

36. Matthews DE, Geissler WB. Arthroscopic suture fixation of displaced tibial eminence fractures. Arthrosc J Arthrosc Relat Surg Off Publ Arthrosc Assoc N Am Int Arthrosc Assoc 1994;10(4):418-23.

37. Padulo J, Oliva F, Frizziero A, Maffulli N. Muscles, Ligaments and Tendons Journal - Basic principles and recommendations in clinical and field Science Research: 2018 update. Muscles Ligaments Tendons J 2018;8(3):305-7. 